\title{
GUILLERMO JÜNEMANN: FILÓLOGO Y CRÍTICO LITERARIO DE LA IGLESIA DE CONCEPCIÓN-CHILE
}

\author{
Guillermo Jünemann: Philologist and Literary Critic from the Church of Concepción-Chile
}

\author{
Pablo Uribe UlloA ${ }^{1}$ (1)
}

\begin{abstract}
Resumen
El presente texto es un acercamiento a la figura desconocida del sacerdoteGuillermo Jünemann. Se muestra el aporte que realizó desde la Iglesia de Concepción entre finales del s. XIX y primera mitad del s. XX, llegando a su obra cumbre que fue la traducción de la primera Biblia en español realizada en América Latina, basándose en la versión griega de la Septuaginta. Los resultados de la investigación muestran quién fue el Padre Jünemann, cuál fue el contexto histórico y eclesial donde desarrolló su ministerio pastoral, qué aportes significativos realizó en la Iglesia de Concepción. Se ha seguido la metodología de análisis documental, realizando una consulta atenta a fuentes bibliográficas de primera mano que corresponden a textos manuscritos como también editados del Padre Jünemann (fuentes), más unos pocos estudios existentes sobre el personaje (bibliografía especializada) y otros textos más generales (bibliografía general). Este trabajo aporta a una línea de investigación poco explorada sobre un personaje ignorado por la historia de la Iglesia contemporánea chilena y latinoamericana.
\end{abstract}

Palabras clave: Jünemann; Biblia; Crítica Literaria; Iglesia; Concepción; Análisis Documental; Septuaginta.

1 Licenciado y Magíster en Teología Bíblica por la Universidad Pontificia de Salamanca, España. Profesor de Sagrada Escritura en el Instituto de Teología de la Universidad Católica de la Santísima Concepción, Chile. Correo electrónico: puribe@ucsc.cl. Este artículo es resultado de una investigación, perteneciente al programa de Doctorado Canónico en Teología de la Facultad de Teología de la Universidad Pontificia Bolivariana, Colombia. 


\section{Summary}

fte following text is an approach to the unknown figure of Father Guillermo Jünemann. It shows the contributionthathemadefrom the church in Concepcion between the end of the XIX century and thefirsthalf of the XX century. fte peak of his work was the first translation into Spanish of the Bible done in Latin America, and based on the Greek version of the Septuagint. fte results of the research show who was Father Jünemann, as well as thehistorical and ecclesial context wherehe developed his pastoralministry, and theimportantwork he did at the church in Concepción. It follows the methodology of documentry analysis, doing an attentive inquiry intofirsthand bibliographic sources, which corresponds to FatherJünemann's manuscripts and edited writings (sources). In addition to some other existing studies of the character (specialized bibliography) and other more general texts (general biography). ftis work contributes to a rarely explored line of inquiry about a characterignored by the history of the contemporary Catholic Church in Chile and in Latin America.

Keywords: Jünemann; Bible; Literary Criticism; Catholic Church; Concepción; Documentary Analysis; Septuagint.

\section{Metodología empleada}

Este artículo ha seguido el método documental entendido como "la captación por parte del investigador de datos aparentemente desconectados, con el fin de que a través del análisis crítico se construyan procesos coherentes de aprehensión del fenómeno y de abstracción discursiva del mismo, para así valorar o apreciar nuevas circunstancias" (Botero, 2003, p. 111). Poco conocida es la figura del Padre Jünemann. No hay estudios suficientes que nos permitan ahondar en su biografía y aporte. Sin embargo, tenemos fuentes directas -poco estudiadas- que son verdaderas joyas para reconstruir su vida y su labor literaria. Por tanto, el método documental nos ha parecido el más adecuado para este trabajo porque se ajusta a nuestro objeto de estudio y, de esta manera, permite validar nuestros resultados. YaMontoya (1976), hace muchos años, en Medellín. decía que este tipo de investigación "se realiza en contacto directo con los documentos y en el recinto cerrado donde éstos se encuentran" (p.3).

Se ha realizado una consulta atenta a fuentes bibliográficas de primera mano que corresponden a textos manuscritos como también editados del Padre Jünemann (fuentes). Estos documentos fuentes se encuentran principalmente en la biblioteca del Seminario Metropolitano de Concepción-Chile, donde hemos colaborado para organizar la colección "fondo Guillermo Jünemann" que reúne 34 cuadernos manuscritos, 9 artículos, 4 cartas y 21 libros. En segundo lugar, hemos rastreado los pocos estudios actuales existentes sobre el personaje (bibliografía especializada), todo ello complementado con un tercer grupo de textos más generales (bibliografía general). No se ha considerado una aproximación bibliométrica porque se aleja del objeto de estudio. 


\section{Análisis de los resultados}

\section{Rasgos biográficos del Padre Jünemann.}

El 28 de mayo de 1855 nació, en la ciudad alemana de Welwer de Vestfalia, Guillermo Jünemann Beckschaefer. Sus padres fueron Federico Jünemann y Cristina Beckschaefer:

Él, un sajón típico: erguido, pálido, de poderosa barba rubia, austero en sus costumbres, de gravedad sazonada por el humor, de corazón fuerte, aunque muy blando.

Ella, vestfaliana no menos típica; pequeña; carirredonda, rosada, de graciosa viveza física e intelectual, incansable y amena conversadora; de corazón femenino, pero capaz de la mayor firmeza y de grandes energías. (Jünemann 1939, p.6)

A la edad de 8 años, junto a sus padres y a sus tres hermanos menores, emprendió un viaje en barco de cuatro meses y medio. Cruzó el Atlántico, pasó por el Cabo de Hornos hacia el Pacífico, llegando a Ancud, Chile. Así, en el año 1863, se estableció en Puerto Montt, sur de Chile, a 96,4 km de Ancud y desde allícomenzó toda una vida enraizada "allá, allá, muy lejos, al otrolado delocéano, al fin del mundo" (Jünemann, 1939, p. 17). No sin dificultad aprendió la lengua nativa de los chilenos, se inició en el latín y, posteriormente, en el griego. Su primera etapa escolar la desarrolló en Puerto Montt, -durante cinco años-bajo la tutoría delsacerdotejesuita JoséZeitlmeier, dequién aprendiólas primeras lecciones de latín. Siempre guardó un especial afecto por este, su primer maestro: "Padre fue para mí, desde entonces, el padre José" (Jünneman, 1939, p.43). Otro barco lo dirigió desde Puerto Montt a Valparaíso, con destino final Santiago. Emprendió, así, su segundo ciclo de estudios en el Colegio Superior San Ignacio, dela compañía de Jesús. No fue fácil la vida de estudiante en Santiago, por su proveniencia humilde del sur de Chile. Pero gracias a su dedicación, prontamente se destacó en lenguas clásicas, y terminósu ciclo de estudios humanísticos con honores. Se cuenta que el mismo rector de la Universidad de Chile, Ignacio Domeyco "le prendió al pecho cuatro medallas de plata, el más alto estímulo establecido" (Naranjo Urrutia 1992, p. 10). En 1872 viajó a Concepción, invitado por el Obispo Hipólito Salas, a continuar sus estudios en el Seminario Conciliar. Estando ya en Concepción, Monseñor Salas le pidió que fuera su secretario y le encargó tareas de redacción de textos en latín para la Santa Sede. Después de 8 años de formación en el Seminario Conciliar de Concepción, fue ordenado sacerdote convirtiéndose, el 18 de marzo de 1880, en el “Padre Jünemann". ${ }^{2}$ Prontamente, el joven sacerdote se convirtió en profesor y, más tarde, rector del Seminario Conciliar.

2 S. Millar Soto plantea que Jünemann fue sacerdote jesuita. Sin embargo, no hemos encontrado evidencia suficiente para tal afirmación. Probablemente, la tesis de Millar Soto se sustenta en la formación de Puerto Montt y Santiago, donde siempre estuvo ligadoa la compañía deJesús. Perolas fuentes queconsultamos afirmansu ordenación en Concepción, en el contexto delSeminario Conciliar y por manos del obispoSalas. Cf. S. MillarSoto, en: https:/ / www.ellanquihue. cl/prontus4_nots/site/artic/20050215/pags/20050215231325.html 
Siempre vinculado al ámbito académico, fue perfeccionando su erudición en filología helenística. Llegó a publicar más de una veintena de libros, destacándose una traducción de La Ilíada de Homero y su magna obra: La Sagrada Biblia. Versión de la Septuaginta al español. ${ }^{3}$

Murió en Tomé, en 1938, dejando dos obras póstumas: Mi camino, y su traducción de la Biblia.S. Millar Soto (2005). En un artículo, el au tor concluye diciendo: "Nunca su fecunda y valiosa labor ha sido difundida o conocida en su Puerto Montt que tanto amóy admiró, lo que es un oprobio para nuestra identidad cultural" ${ }_{4}^{4}$ Por otra parte, A. González (2011) afirma que al "políglota de nueve lenguas, casi nadie lo recuerda hoy" (p. 69).

\section{La Iglesia deConcepción}

"La historia pastoral dela arquidiócesis ha estado marcada por un constante impulso misionero y entrega evangelizadora, en la que reconocemos la moción del Espíritu Santo". Con estas palabras, el arzobispo actual de Concepción, Mons. Fernando Chomali Garib (2016, p.3), describe lo que es la arquidiócesis de la Santísima Concepción. Con más de 450 años de historia y fundada el 22 de marzo de 1563 por el Papa Pío IV mediante la Bula Super specula militantes Ecclesiae, a petición del rey Felipe II de España, con el nombre de Imperial. El primer obispo fue Don Antonio de San Migue Avendaño y Paz, ofm. En 1600 , los indígenas araucanos, en constantes batallas con los españoles, destruyeron la Imperial hasta sus cimientos, huyendo sus habitantes hacia el norte, es decir, a Concepción (Diócesis de Concepción, 1926, p.IV). Desde aquel momento, la sede episcopal se trasladó a Concepción, quedando provisionalmente

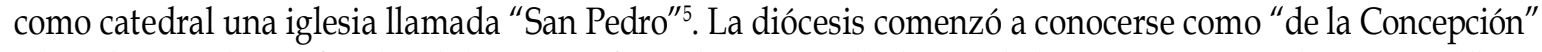
y los obispos, hasta finales del s. XVIII, firmaban como "Obispo de la Concepción y de Imperial". En 1751, un violento terremoto destruyó la ciudad (Penco), lo que obligó a trasladar la diócesis al valle de la Mocha o de Rozas, es decir, a la actual Concepción. En 1835, otro terremoto destruyó la catedral y la nueva catedral, construida en el mismo terreno, fue consagrada bajo la advocación de la Inmaculada Concepción de María. Desde ese momento, la diócesis -convertida en arquidiócesis desde 1939- recibió el nombre de la Santísima Concepción 6 .

La vida adulta del padre Jünemann transcurrió, entre finales del s. XIX y principios dels. XX, en esta diócesis, y en un país marcado por un liberalismo y laicismo que comenzaba aimponerse (SánchezGaete, 2011). El historiador Andrés Medina (1997) explica que en el s. XIX, en Chile:

3 Sobre su actividad literaria volveremos más adelante, en el apartado tercero: "la obra editorial del Padre Jünemann".

4 Cf.https://www.ellanquihue.cl/prontus4_nots/site/artic/20050215/pags/20050215231325.html

5 Se trata de una iglesia situada en Penco, ciudad donde se emplazó la diócesis antes de su ubicación actual en la ribera del río Bíobío.

6 Actualmente, la arquidiócesis de la Santísima Concepción posee una extensión territorial de 11.400 km2, una población de 1.367.144 habitantes, con 55 parroquias, 60 presbíteros seculares ( 56 chilenos y 4 extranjeros), más 8 presbíteros seculares de otras diócesis ( 3 chilenos y 5 extranjeros), y 56 diáconos permanentes. 
Se produce un quiebre entre un Estado autoritario, conservador y de abierto compromiso con la Iglesia a través del patronato existente entre 1830 y 1872, con un nuevo Estado, liberal y de tendencia laica que busca disminuir la influencia que el catolicismo había ejercido en el seno social. (p.12)

Una destacada figura de este tiempo fue monseñor José Hipólito Salas y Toro, (1812-1883), obispo de Concepción entre los años 1854 al 1883. Reabrió el Seminario Conciliar, participó como perito en el Concilio Vaticano I (Etchegaray Cruz, 1963), construyó una nueva catedral y realizó misiones entre los mapuches. Buen administrador episcopal y organizador de la curia: "En definitiva, fue un hombre que con su largo gobierno episcopal junto a sus innegables capacidades puso a Concepción en el sitial que le correspondía" (Moreno Jeira, 2011, p.41).

Por otra parte, a comienzos del s. XX, el panorama del clero chileno era el siguiente?:

A las órdenes dela iglesia chilena hallase un clero secular compuesto de 687 sacerdotes, un clero regular que consta de 1421 religiosos y, ayudando á ambos en las obras del apostolado, 33 congregaciones de mujeres cuyos miembros alcanzan... á 2283(Emeth, 1910).

La industria comenzó a cimentarse en las ciudades de la diócesis. Así, en 1852 se inició el desarrollo de la explotación minera de carbón en Lota y Coronel, con Matías Cousiño y Federico Schwager, respectivamente; en 1913, en Tomé, surgió la Sociedad Nacional de Paños y, en Penco, se fundó la Fábrica Nacional de Loza (FANALOZA) en 1927 (Astorquiza \& Galleguillos, 1952; Cartes, Luppi \& López, 2012; Márquez Ochoa, 2018). El Padre Jünemann ejerció su acción pastoral en Schwager. Recuerda aquella experiencia de la siguiente manera: "Corre el año 1897. Estoy a cargo de mi antigua parroquia de Coronel, pequeña cuidad, triste como pocas, como pocas pobre de religión, pobrísima, casi falta, de hogares ni medianamente cultos" (Jünemann, 1939, p.366). Por tanto, estas ciudades industrializadas serán el contexto de su ministerio sacerdotal que se divide entre el cultivo intelectual y la realidad de la pobreza de los trabajadores, especialmente el minero del carbón y su entorno.

\section{La obra editorial del padre Jünemann}

Si tuviéramos quecaracterizar el trabajo del padre Jünemann, deberíamos situarlocomo filólogo y crítico literario. En el contexto de la Iglesia de Concepción de la primera mitad del siglo XX, es un fenómeno único. Su labor literaria alcanzó el apoyo de la reconocida editorial Herder, de Alemania, lo que confirma su talento y pericia en el arte de la traducción y dominio de las letras. Lo poco que se conoce a Jünemann en el mundo académico es por su traducción de la Biblia. Su obra de escritor es amplia, pero sus textos no están al alcance de todos, ya sea por la restricción del tiraje, porque ya hace mucho tiempo en que se escribieron o por las limitadas capacidades de las editoriales e imprentas locales, donde muchas de sus obras vieron la luz.

7 Deaquí en adelante citaremos los textos antiguos tal cualse encuentran enlas fuentes directas quehemos consultado, manteniendo la forma de escribir de aquellos años. 
El estudio más completo que existe sobre el padre Jünemann presenta un elenco de 15 obras, incluyendo 4 inéditas, a saber: "Clio", "Susana", "Ensayos" y "Biblia de Jünemann”, que hasta esa fecha noestaba publicada ${ }^{8}$. Sin embargo, hemos encontrado un registro mayor de obras, lascuales consultamos directamente, y que hoy se encuentran -la mayoría de ellas- en el fondo Jünemann, en la biblioteca del Seminario Metropolitano de Concepción. En orden cronológico reconstruimos el siguiente listado de treinta y cuatro obras":

- Carta a Marcelino Menéndez Pelayo, 1893.

- Carta a Marcelino Menéndez Pelayo, 1894.

- Carta a Marcelino Menéndez Pelayo, 1895.

- Historia de la literatura, 1898.

- Carta a Marcelino Menéndez Pelayo, 1900.

- Traducción de la Ilíada, 1902.

- "De suicidio y suicidios", 1906.

- "Proceso secular", 1906-1907.

- "Anticlericalismo francés", 1907.

- "Pensamiento sobre las indulgencias", 1907.

- "Hacia la libertad", 1907.

- "Modernísticas", 1909.

- Antología universal de los mayores genios literarios, 1910.

- Paulina de Mallinckrodt y su obra, 1911.

- Antología escolar latina, 1912.

- Historia de la Literatura Española y antología de la misma, 1913.

- Apuntes exegéticos sobre el $4^{\circ}$ evangelio, la epístola a los Romanos, Salterio y Génesis, 1915.

- Historia elemental de la Literatura, 1917.

- Conversaciones sobre Alemania y la Gran Guerra,1918.

- “Eterno", 1921.

- "Rebeldías vencidas", 1921.

- Estética Literaria, 1924.

- Literatura Universal, 1926.

- "Algo de prehistoria del México actual", 1928.

- Nuevo Testamento, 1928.

- Historia y antología de la literatura española,1928.

- Teresa. Las maravillas de Konnersreuth,1929.

8 El estudio es un verdadero artículo de 34 páginas, firmado por Alfonso Naranjo Urrutia, que circuló un par de años antes de la publicación de la Biblia como folleto promocional de esta. No tiene fecha ni lugar de publicación, pero lo más probable es que haya sido impreso en Santiago, ya que el autor figurajunto a Gustavo Leiva, como uno de aquellos a quienes hay que hacerle reserva de la Biblia, ambos con domicilio en Santiago de Chile. En la contraportada del folleto selee: “Este folleto que ha llegado a sus manos, es de distribución gratuita, para quienes hagan la reserva de un ejemplar de la Biblia de Jünemann. Para los que no hagan reserva, les rogamos ayudar con un aporte, el cual puede enviarse en un cheque o pedir que vaya a retirarse. Indique dirección". (Naranjo Urrutia, 1992).

9 Los títulos en cursiva son libros, los que están entre comillas, artículos y los títulos en redonda son cartas. 
- Elegíacas, 1930.

- Azucena. A la memoria de la Srta. Salomé Villalobos Ávila (11 febr. 1865-22 febr. 1932), 1932.

- Semblanza de mi madre. Cristina Beckschaefer de Jünemann, 1939.

- Mi Camino, 1939.

- Isaías, 1984.

- Job y Salmos, 1986.

- La Sagrada Biblia. Versión de la Septuaginta al español, 1992.

Toda la obra del p. Jünemann la podemos clasificar como literatura, traducciones, biografías y ensayos. Sobre estos grandes grupos iremos dando una caracterización. En este caso, no seguiremos un orden cronológico, sino temático.

\section{Literatura}

La mayor parte de su producción académica corresponde a obras relacionadas con la literatura. En 1898, publicó la Historia de la literatura, obra de 303 páginas, publicada en Concepción. Al poco tiempo apareció una segunda edición, "cuidadosamente revisada", como reza su epígrafe, realizada en Friburgo, Alemania, por B. Herder. Como su nombre lo indica, se hace un recorrido por la historia universal, destacando las principales obras. En la primera parte denomina al Pentateuco y otros libros bíblicos como el: "libro 1: Literaturas antiguas, primera parte: literaturas orientales, sección I "Literatura hebrea, la Sagrada Escritura" (pp. 9-28). La cita anterior demuestra la claridad que tenía el p. Jünemann en aquellos años, en torno a la riqueza literaria que la Biblia encierra. Sobre la base de la misma obra, publica en 1910, con Herder, la Antología universal de los mayores genios literarios, con capítulos dedicados a las literaturas hebrea, griega, latina, española, portuguesa, francesa, italiana, alemana, inglesa y otro sobre himnología medieval. Comienza la literatura hebrea con el pentateuco y, en la introducción, plantea, con gran convicción -de acuerdo al estado del arte de la época-, lo siguiente:

El más antiguo é importante libro histórico, no sólo de la Biblia, sino del mundo entero, es el Pentateuco (ó sea, cinco libros en un volumen), que consta de Génesis, Éxodo, Levítico, Números y Deuteronomio. Fuéescrito, álo menos encuantoála substancia, entrelossiglos XVIy XV antesdeJ.C., por Moisés, el célebre caudillo del pueblo hebreo (p.1).

En 531 páginas va seleccionando textos de todos los periodos históricos, insistiendo en mostrar la Biblia como parte del patrimonio literario de la antigüedad. Conun fin pedagógico, publica en 1912 la Antología escolar latina. Al comienzo del libro se lee: "con inclusión de lo que se exige en el examen de latín para el Bachillerato en Chile" (p. 2). Contiene dos partes, la primera, con textos en prosa, donde selecciona fragmentos de César, deSalustioy de Cicerón. La segunda está dedicada a textos poéticos, con selecciones de Horacio, de Virgilio, de Ovidio y un apéndice con himnos eclesiásticos. Carece de introducción o información sobre los textos. En esta misma línea de selección de textos -para ponerlos al alcance del gran público-, edita, con Herder, al año siguiente, Historia de la Literatura Española y antología de la misma, obra de 268 páginas. Le sigue en 1917 una pequeña obra de 97 páginas, editada en Concepción, Historia elemental de la Literatura, un resumen de obras anteriores. En 1924 publica, con Herder, la voluminosa obra de 417 páginas Estética Literaria. Dos años más tarde, refunde, en una sexta edición mejorada, de 339 páginas -de nuevo con Herder-, la Literatura Universal. En sus primeras páginas se indica que la 
edición cuenta con una lámina frontispicio y sesenta y dos grabados en el texto. Es recomendada por el ministerio de instrucción pública de España y adaptada como texto de los institutos oficiales de Venezuela. En 1928 publica una tercera edición de Historia y antología de la literaturaespañola, de 285 páginas, otra vez con Herder.

En otros géneros literarios Jünemann publicó dos obras curiosas: unas "conversaciones" y una pequeña obra poética. La primera, de 1914, reimpresa en 1915, en Concepción, y con una segunda edición en 1918, se llama Conversaciones sobre Alemania y la Gran Guerra. Trata del tema de la guerra mediante un diálogo entre 2 personajes, J. y A. No hay ninguna explicación de los personajes, al parecer dos hombres, buenos amigos., Al final del libro, aparece $\mathrm{M}$, que es una mujer:

"M.- ¿interrumpo o estorbo?

J.-Nilouno nilootro, señorita. Al contrario, el tema es para queusted se tercieen él.

M.- ¿De qué tratan ustedes?

J.- En este momento, de economía doméstica y de cuán bien la entiende el hogar alemán, la mujer alemana. (p.92)

Elégicas es la única obra poética que hemos encontrado del autor. Publicada en Concepción, en 1930, consta de 185 páginas. Obra interesante que se divide cronológicamente en textos anteriores a 1930 y textos del año 1930. Además, al final, incluye una obrita llamada "San Pelayo (Balada)", escrita en Concepción, el 8 de octubre de 1926.

\section{Traducciones}

La labor de traductor la tenemos expresada en sus dos grandes obras: La Ilíada y la Biblia. En 1902, en Concepción, publica la Traducción de la Ilíada, de 547 páginas. Al comienzo se lee: "La Ilíada de Homero. Esplend or la tierra y las estrellas. Traducción directa fidelísima y crítica por G. Jünemann". Al traducir la Ilíada, consulta a Marcelino MenéndezPelayo:

Por el correo de hoy envio á Vd. la traducción del primer canto de la Ilíada, para que, en algun momento completamente libre, tenga la bondad de echarle una ojeada y de decirme, con toda libertad, el juicio que el trabajo le merezca y de hacerme las observaciones que le sugiera. Lo único quele puedo decir, es quela traducción es fidelísima, y literal en toda la extensión de la palabra $(1894)^{10}$.

Notenemos las respuestas deMenéndezPelayo-si es que respondió a alguna de sus cartas-. Locierto es que Jünemann tenía en mente hacer una traducción a dos columnas, una literal y otra más libre. Pero finalmente ofreció solo la traducción literal "fidelísima y crítica" , como él mismo lo afirma. Relacionada con las traducciones hay una pequeña obra bíblica Apuntes exegéticos sobre el $4^{\circ}$ evangelio, la epístola a los Romanos, Salterio y Génesis, de 164 páginas. Solo hemos tenido acceso a la segunda edición, de 1915, publicada en Concepción. En esta obra, el autor da una razón muy importante sobre la consideración positiva del texto bíblico griego sobre el hebreo para el Antiguo Testamento. En la p. 7 se lee:

10 Se ha mantenido el texto original, que difiere a la escritura del español actual. Poseemos 4 cartas a Menéndez Pelayo, las tres primeras envidas desdeSan Javier en los años 1893,1894,1895y laúltima, ya desde Concepción, en 1900. 
Prevalece la versión grandemente sobre el texto masorético; que es de origen incierto; relativamente moderno y dimanado al parecer de un solo códice ${ }^{11}$. Sixto $\mathrm{V}$ (después dehechos inmensos estudios críticos de toda suertedecódices) fijó el texto griego o Septuaginta, tomando por base elcódice Vaticano, el reconocidamente mejor supliendo, de los otros manuscritos principales, las lagunas.

Esta parece ser la razón-además de su amplio dominio del griego helenístico-dehaber traducido la Biblia completa desde la versión de los LXX y no del tex to hebreo masorético, como era y es actualmente la tendencia entre los traductores bíblicos. Jünmann realizó su traducción bíblica entre1921 y 1928. Monseñor Antonio Moreno Casamitjana (1992) señala el objetivo de aquella aventura que le significó 7 largos años de trabajo: "Su intención fue dar una versión lo más literal posible de la versión de los LXX, queapreció, como texto apostólico, por encima del texto hebreo masorético". (1992, p.19) Para Jünemann, esta será la gran obra de toda su vida. Él mismo cuenta cómo se dio a esta titánica tarea:

¿El deseo de verter la palabra divina con la misma fidelidad, el mismo respeto, el mismo amor que había vertido tanta palabra humana?, ¿verterla de modo que no tuviese yo que avergonzarme delante de Dios por irrespetuoso, ni delante del idioma español, ruborizándome de rigidez y pobreza?

Así pensaba. Pero aún no cogía la pluma.

Cógila de repente. Y fue de esta manera:

Un día me dice casi de improviso una niña: 'cuando abro el evangelio, no sé lo que me pasa: me olvido de todo; me parece que no estoy aquí'.

Y yo: 'cuánto más gozaría Ud., si lo leyese exactamente traducido; no tan mal como lo está'.

Ella: Y ¿por qué no lo traduce bien Ud.; ya que escribe tantas otras cosas? Yolecopio.

Yo: Ud. sabe que mi editor (B. Herder) y Alemania son actualmente, como si no existieran. ¿Quién me lo imprime?

Ella: Yo le ayudo a costear la edición...'

Añadiréque distaba ella mucho de ser rica; y no tengo ya necesidad de decir queel mismo día cogíla pluma, y no la soltaré hasta que termine mi trabajo, si Dios antes no me la quita de la mano.

Esta es la génesis de mi versión de la biblia". (1939, p.533-534)

No logró ver la Biblia completa editada, aunque ya en 1928 tenía la traducción completa realizada. Sí publicó el Nuevo Testamento, él mismo 1928, en Concepción, agregando el subtítulo: "Versión directa del griego según los mejores códices (vaticano, sinaítico, alejandrino) y sus ediciones" (p.1); y una advertencia: "Tanfielhetraducidola palabradivina enlengua española, quenotengaqueavergonzarme, yo, anteDios, de irreverente; ni ella ante los hombres, de pobre" (p.3). Esta versión del NT consta de 581 páginas. La traducción del Antiguo Testamento permaneció en 34 cuadernos manuscritos esperando su publicación completa, que solo ocurrió en 1992, bajo el título: La Sagrada Biblia. Versión dela Septuagintaal español. Anterior a la Biblia editada, se publicaron, parcialmente, tres libros del AT, en dos volúmenes: Isaías y Job y Salmos. Isaías (1984), consta de110 páginas y junto a Job y Salmos (1986), explica a los lectores, en una

11 Talvez estépensando en el Codex de Leningrado, datado hacia el1008-1009d.C. Estecódice manuscrito es el que presenta, de manera completa y mejor conservada, la Biblia hebrea. Se encuentra desde 1863 en la Biblioteca Nacional de Rusia, en San Petersburgo, bajo la catalogación B19a. Actualmente sigue siendo un gran testimonio del texto masorético y es la base dela conocida edición crítica dela Bibia Hebraica Stuttgartensia (BHS) que se usa para todas las traducciones modernas del Antiguo Testamento. 
introducción al libro, tomada de la sexta edición de su Literatura Universal, el origen de esta traducción de Isaías:

Nopudiendoeditar, porfalta de dineros, toda la Biblia, según la Versión española deGuillermoJünemann B., nos place ofrecer a los estudiantes y amantes dela Palabra Divina, esta edición del profeta Isaías, esperando que Dios nos conceda la gracia de editar pronto otra parte de esta Versión, ya sea otro profeta u otro de los libros sagrados.

Las notas, al margen, son delos santos padres y deautorizados exégetas católicos y sirven para esclarecer el texto. $(1984$, p.9)

Firma Ambrosio Villa Echeverría, editor, en Angol, en 1983. El libro no tiene notas al margen, solo a pie de página. Al cotejarlo con el Ms. Cuaderno 25, y con la Biblia es perceptible que se trata de un mismo texto, que no indican de dónde han sido sacados. Así, por ejemplo, al final del capítulo 3, hay una nota, específicamente en Is 3,22, donde se lee: "y los cendales los para la casa y los diáfanos lacónicos" (Biblia Jünemann, 1928). Aquí se incorpora una nota al pie que indica "túnica corta lujosa espartana" (Biblia Jünemann, 1928). Job y Salmos es de 1986, tiene 208 páginas y fue editado también por Ambrosio Villa E. Incorpora, también, la frase que Jünemann usó para el Nuevo Testamento: "versión directa del griego, fidelísima y crítica según los mejores códices". Al comienzo se indica que el libro es de1985 y al final del libro se indica que es de 1986. Finalmente -como hemos mencionado más arriba-, en 1992, los ex alumnos del Seminario publicaron, en Santiago de Chile, la Biblia completa. En la primera página se lee: "La Sagrada Biblia. Versión de la Septuaginta al español. Pbro. Guillermo Jünemann Beckschaefer. Versión directa delGriego, Hebreo y Arameo. Según los mejores códices: Vaticano,Sinaítico, Alejandrino y sus mejores ediciones". La alusión a una versión directa del "hebreo y arameo" no corresponde a una traducción basada en laSeptuaginta. Jünemann no poneesto en suscuadernos manuscritos y es, a nuestro juicio, un error de los editores. Jünemann sí pone un colofón, el día en que terminó su traducción, el 28 de octubre de 1928: "María, tú que conservabas, en tu corazón, la palabra divina, da una mirada a esta mi versión española de ella, para que se conserve y propague, llevando a muchas almas y que solo ella puede dar". Del NT se ha tomado de la versión editada en 1928 por Jünemann. Además de usar la versión de los LXX como base de su traducción, Jünemann incorporó dos libros apócrifos: El Libro III de Esdras y el Libro III de los Macabeos, que sí están en el llamado canon alejandrino de los LXX y no así en el canon palestinense hebreo. Jünemann los pone al final del Antiguo Testamento.

\section{Biografías}

Un tercer grupo de sus obras pertenecen al género biográfico. La más importante es, sin duda, Mi Camino, publicadaen 1939. Elsubtítulo de esta autobiografíaaclara su orientación:" apuntes autobiográficos sobre mi labor crítica" (p.1). Se trata de un voluminoso texto de 554 páginas, dividido en 310 pequeños capítulos o temas. Una segunda biografía importante es Azucena. A la memoria de la Srta. Salomé Villalobos Ávila (11 febr. 1865-22 febr. 1932), publicada en Concepción en 1932, con 127 páginas. El libro contiene una nota que indica "circulación estrictamente privada" y el punto 1 -de 147- lleva por título: No para todos:

Escribo sólo para los amigos y para los de espíritu bastante recto y limpio para vislumbrar al menos que pueda existir entre hombre y mujer amistad sincera, íntima y pura.

Paralos queno alcanzan a comprender tal afecto, sino que todo querer lo creen carne, sensualidad y corrupción; para esos, líbreme Dios de escribir y de darles aquí una sola mirada: me rebajaría y pareceríame 
ofender la memoria de aquella cuyo nombre deseo perpetuar entre los que la han querido y se complacerán en mirar su imagen. (p.3).

Entre 1911 y 1939 publica, con Herder, Paulina de Mallinckrodt, la cual consta de 168 páginas; Teresa. Las maravillas de Konnersreuth, en Concepción, 48 páginas y una obrita muy sentida: Semblanza de mi madre. Cristina Beckschaefer de Jünemann, escrita y publicada en Padre de las Casas por la impronta San Francisco con 41 páginas.

\section{Ensayos}

Finalmente, presentamos unos artículos publicados en La Revista Católica, deSantiago deChile, entre 1906 y 1928. Estos textos, más que abordar cuestiones propiamente teológicas, van reflexionando, a modo de ensayos, sobre diversas temáticas de la época, como el diálogo entrelafey el modernismo. Así, tenemosDe suicidio y suicidios (1906), Proceso secular (1906-1907), Anticlericalismofrancés (1907), Pensamiento sobre las indulgencias (1907), Hacia la libertad (1907), Modernísticas (1909), con 6 entregas, Eterno (1921), Rebeldías vencidas (1921), Algo de prehistoria del México actual, (1928).

\section{Conclusión}

Alcomienzo deeste estudio formulamos unas preguntas queahora, a modo de conclusión, resumiremos deacuerdo a todolo anteriormente desarrollado. 1. ¿Quién fueGuillermoJünemann? Sacerdote, hombre de fe, amante de las letras, del mundo clásico, de las lenguas y de la estética, un sacrificado inmigrante alemán que llegó de niño para quedarse definitivamente en Chile. 2. ¿Cuál fue el contex to histórico y eclesial en el que le tocó desarrollar su ministerio pastoral? La Iglesia chilena de mitad del s. XIX y principios del XX, marcada por el contexto de la independencia, posteriormente por el modernismo y el laicismo chileno. 3. ¿Qué aportes significativos realizó el padre Jünemann a la Iglesia de Concepción? Su aporte fue eminentemente intelectual, pues era poco cercano a sus estudiantes, los seminaristas "Se podría decir que Jünemann creía que su morada estaba en el Olimpo y no en el mundo común de todos nosotros" -refiere un ex alumno. Otro dirá que: "Su carácter hosco, muy amigo de la crítica, apasionado en lo intelectual, poco accesible, demasiado docto". Desarrolló labores pastorales, fue capellán y párroco, pero se dedicó pastoralmente al estudio y a la formación de los estudiantes del seminario. Así, comprendió el sacerdocio como un ministerio de la más alta responsabilidad en la Iglesia. Sus prédicas, muy bien elaboradas, verdaderas catequesis, colaboraban con ese afán formador. Ciertamente un autor desconocido que hizo un gran aporte a la iglesia latinoamericana en su labor de filólogo y crítico literario desdela diócesis deConcepción-Chile.

\section{Referencias}

Astorquiza, O. \& Galleguillos, O. (1952). Cien años del carbón de Lota. 1852-1952. Santiago: Compañía Carbonífera e Industrial de Lota.

Botero Bernal, A. (2003). La metodología documental en la investigación jurídica: alcances y perspectivas. Opinión Jurídica (2), 109-116. 
Bravo, A. (2007). Anunciaré tu fidelidad. Anales de Teología UCSC, 9 (1), 7-40.

Bravo, A. (2013). Antonio Moreno Casamitjana: profeta de nuestro tiempo. Anales de Teología UCSC, 15 (2), 495-515.

Campos Harriet, F.(1980). Historia de Concepción 1550-1970. Santiago: Editorial Universitaria.

Cartes, A., Luppi, R. \& López, L. (2012). Bellavista Oveja Tomé. Una fábrica en el tiempo. Concepción: Ediciones Universidad San Sebastián.

Chomali, F. (2016). Volver a Jesucristo para renovar la Iglesia. Constituciones Post Sinodales. Concepción: Arzobispado de la Santísima Concepción.

Diócesis de Concepción. (1922). Consuestas de la Iglesia Catedral de Concepción Chile. Santiago: Imprenta de San José.

Elliger, K. \& Rudolph, W.(Eds). (1997). Biblia Hebraica Stuttgartensia. Stuttgar: Deutsche Bibelgesellschaft.

Emeth, O. La Iglesia católica en Chile. Desde 1810 a 1910. Revista Zig-Zag, 252 (1910).

Etchegaray Cruz, A. (1963). “Mons. José Hipólito Salas enel Concilio Vaticano I. Historia (2), 134-167.

Gazmuri Riveros, C. (2006-2009). La historiografía chilena (1842-1970). 2 Vol. Santiago: Aguilar Chilena Ediciones.

González Schaín, A. (2011). Jünemann: la primera Biblia chilena y de Latinoamérica. Revista de Ciencias Religiosas, 19 (2), 61-71.

Jünemann, G. (1894). CartaaMarcelinoMenéndez Pelayo.SanJavier, Chile19demarzo1894.

Jünemann, G. (1895). Cartaa Marcelino Menéndez Pelayo. San Javier, Chile18 deenero1895.

Jünemann, G. (1898). Historia de la literatura. Concepción: Imprenta y encuadernación del Águila.

Jünemann, G. (1900). Carta a Marcelino Menéndez Pelayo. Concepción, Chile 17 de agosto 1900.

Jünemann, G. (1902). Traducción dela Ilíada. Concepción: Litografía eimprenta Concepción B. Paschen W.

Jünemann, G. (1906). De suicidio y suicidios. La Revista Católica (6), 676-680.

Jünemann, G. (1907). Anticlericalismo francés. La Revista Católica (7), 125-129.

Jünemann, G. (1907). Hacia la libertad. La Revista Católica (7), 818-823.

Jünemann, G. (1907). Pensamientos sobre las indulgencias. La Revista Católica (7), 494-497.

Jünemann, G. (1907). Proceso secular. La Revista Católica (11-12), 775-783; (13-14), 808-818.

Jünemann, G.(1909). Modernísticas. La Revista Católica (9),598-608;740-749, 834-840;911-916;(17)110-122, 285-294.

Jünemann, G. (1910). Antología universal de los mayores genios literarios. Friburgo: Herder \& Cía.

Jünemann, G. (1911). Paulina de Mallinckrodt y su obra. Friburgo: Herder.

Jünemann, G. (1913). Historia de la Literatura Española y antología de la misma. Friburgo: Herder \& Cía.

Jünemann, G. (1915). Apuntes exegéticos sobre el $4^{0}$ evangelio, la epistola a los Romanos, salterio y Génesis. Concepción: Litografía y Tipología José V.Soulodre.

Jünemann, G. (1917). Historia elemental de la Literatura. Concepción: Imprenta Penquista. 
Jünemann, G. (1918). Carta a Marcelino Menéndez Pelayo, San Javier, Chile, 21 de junio 1893.

Jünemann, G. (1918). Conversaciones sobre Alemania y la Gran Guerra. Concepción: Litografía Nacional, Luis Bittner y Cía.

Jünemann, G. (1921). Antología escolarlatina. Concepción: Escuela deartes y oficios deS. José.

Jünemann, G. (1921). Eterno. La Revista Católica, (21), 412-416.

Jünemann, G. (1921). Rebeldías vencidas. La Revista Católica, (21), 357-364.

Jünemann, G. (1924). Estética Literaria. Friburgo: Herder \& Cía.

Jünemann, G. (1926). Literatura Universal. Friburgo: Herder \& Cía.

Jünemann, G. (1928). Algo de prehistoria del México actual. La Revista Católica (28), 134-140.

Jünemann, G. (1928). Historia y antología de la literatura española. Friburgo: Herder \& Cía.

Jünemann, G. (1928). Nuevo Testamento. Concepción: Editorial de la librería Diocesana.

Jünemann, G. (1929). Teresa. Las maravillas de Konnersreuth. Concepción: Editorialdela Librería Diocesana.

Jünemann, G. (1930). Elégicas. Concepción: Editorial de la Librería Diocesana.

Jünemann, G. (1932). Azucena. A la memoria de la Srta. Salomé Villalobos Ávila (11 febr. 1865-22 febr. 1932). Concepción: Imprenta Esmelalda.

Jünemann, G. (1939). Mi Camino. Imprenta San Francisco, Padre de las Casas.

Jünemann, G. (1939). Semblanza de mi madre. Cristina Beckschaefer de Jünemann. Imprenta San Francisco, Padre de las Casas.

Jünemann, G. (1984). Isaías. Imprenta y editorial San Francisco, Padre de las Casas.

Jünemann, G. (1986). Job y Salmos. Editorial San Francisco, Padre de las Casas.

Jünemann, G. (1992). La Sagrada Biblia. Versión de la Septuaginta al español. Santiago: Centro de ex alumnos del Seminario Conciliar de Concepción.

Jünemann, G. Ms. Cuadernos1 detraducción dela Biblia, Concepción.

Jünemann, G. Ms. Cuadernos 10 de traducción de la Biblia, Concepción.

Jünemann, G. Ms. Cuadernos 11 de traducción de la Biblia, Concepción.

Jünemann, G. Ms. Cuadernos 12 de traducción de la Biblia, Concepción.

Jünemann, G. Ms. Cuadernos 13 de traducción de la Biblia, Concepción.

Jünemann, G. Ms. Cuadernos 14 de traducción de la Biblia, Concepción.

Jünemann, G. Ms. Cuadernos 15 de traducción de la Biblia, Concepción.

Jünemann, G. Ms. Cuadernos 16 de traducción de la Biblia, Concepción.

Jünemann, G. Ms. Cuadernos 17 de traducción de la Biblia, Concepción. 
Jünemann, G. Ms. Cuadernos 18 de traducción de la Biblia, Concepción. Jünemann, G. Ms. Cuadernos 19 de traducción de la Biblia, Concepción. Jünemann, G. Ms. Cuadernos 2detraduccióndela Biblia, Concepción. Jünemann, G. Ms. Cuadernos 20 de traducción de la Biblia, Concepción. Jünemann, G. Ms. Cuadernos 21 de traducción de la Biblia, Concepción. Jünemann, G. Ms. Cuadernos 22 de traducción de la Biblia, Concepción. Jünemann, G. Ms. Cuadernos 23 de traducción de la Biblia, Concepción. Jünemann, G. Ms. Cuadernos 24 de traducción de la Biblia, Concepción. Jünemann, G. Ms. Cuadernos 25 de traducción de la Biblia, Concepción. Jünemann, G. Ms. Cuadernos 26 de traducción de la Biblia, Concepción. Jünemann, G. Ms. Cuadernos 27 de traducción de la Biblia, Concepción. Jünemann, G. Ms. Cuadernos 28 de traducción de la Biblia, Concepción. Jünemann, G. Ms. Cuadernos 29 de traducción de la Biblia, Concepción. Jünemann, G. Ms. Cuadernos3 detraduccióndela Biblia, Concepción. Jünemann, G. Ms. Cuadernos 30 de traducción de la Biblia, Concepción. Jünemann, G. Ms. Cuadernos 31 de traducción de la Biblia, Concepción. Jünemann, G. Ms. Cuadernos 32 de traducción de la Biblia, Concepción. Jünemann, G. Ms. Cuadernos 33 de traducción de la Biblia, Concepción. Jünemann, G. Ms. Cuadernos 34 de traducción de la Biblia, Concepción. Jünemann, G. Ms. Cuadernos 4 detraduccióndela Biblia, Concepción. Jünemann, G. Ms. Cuadernos 5 detraduccióndela Biblia, Concepción. Jünemann, G. Ms. Cuadernos6 detraducción dela Biblia, Concepción. Jünemann, G. Ms. Cuadernos 7 detraducción dela Biblia, Concepción. Jünemann, G. Ms. Cuadernos 8detraducción dela Biblia, Concepción.

Jünemann, G. Ms. Cuadernos 9 detraducción dela Biblia, Concepción.

Márquez Ochoa, B. (2018). Cerámica en Penco, industria y sociedad: 1888-1962. Concepción: Ediciones Archivo Histórico de Concepción.

Medina, A. (1997). Monseñor José Hipólito Salas. Obispo de Concepción. Concecpión: Centro Teológico UCSC.

Millar Soto, S. (2005). Jünemann, eximio escritor olvidado. El Llanquihue, 16, febrero 2005.

Montoya Gutiérrez, S. (1976). Procedimiento y técnica de la investigación documental en la Universidad. Medellín: Universidad de Medellín. 
NaranjoUrrutia, A. (1992). La Biblia de Jünemann. ¿Quién es el sacerdote Guillermo Jünemann B.?Santiago.

Sánchez Gaete, M. (Dir.). (2011). Historia de la Iglesia en Chile. Los nuevos caminos: la Iglesia y el Estado. Santiago: Editorial Universitaria.

Straubinger, J. (1947). Guillermo Jünemann, primer traductor de la Biblia en América. Revista Bíblica (45), 73-75.

Straubinger, J. (1947). Guillermo Jünemann, primer traductor de la Biblia en América (continuación). Revista Bíblica (46), 124-127.

UribeUlloa, P.\& Inostroza J.C. (Coords.). (2009). Annuntiaboveritatem tua. Estudiosen homenajea Mons. Antonio Moreno Casamitjana. Concepción: Universidad Católica de la Ssma. Concepción.

UribeUlloa, P.(2011). Pasadoy presentedelos estudios bíblicosen Chile. AnalesdeTeologíaUCSC,13(2),373-395.

Uribe Ulloa, P.(2015). Recepción bíblica de la Constitución Dei Verbum en América Latina. Medellín (162), 283-305. 